Revue internationale P.M.E.

Économie et gestion de la petite et moyenne entreprise

\title{
La gestion des compétences dans la stratégie de croissance d'une PME innovante: le cas Microtek
}

\section{Christian Defélix et Didier Retour}

Volume 16, numéro 3-4, 2003

URI : https://id.erudit.org/iderudit/1008444ar

DOI : https://doi.org/10.7202/1008444ar

Aller au sommaire du numéro

Éditeur(s)

Presses de l'Université du Québec

ISSN

0776-5436 (imprimé)

1918-9699 (numérique)

Découvrir la revue

Citer cet article

Defélix, C. \& Retour, D. (2003). La gestion des compétences dans la stratégie de croissance d'une PME innovante: le cas Microtek. Revue internationale P.M.E., 16(3-4), 31-52. https://doi.org/10.7202/1008444ar
Résumé de l'article

La recherche sur les PME a jusqu'ici mis en lumière les enjeux ressources humaines de la croissance ainsi que l'existence de pratiques de gestion des compétences. Mais, de façon surprenante, ces deux dimensions n’ont pas été croisées jusqu'à présent : que sait-on de l'apparition et du développement de la gestion des compétences dans les PME en croissance ? Le présent article propose un cadre d'analyse et l'applique à une étude de cas longitudinale et approfondie, celle de l'entreprise Microtek. 


\title{
La gestion des compétences dans la stratégie de croissance d'une PME innovante: le cas Microtek
}

\author{
Christian DEFÉLIX \\ Université Joseph-Fourier, Grenoble I
}

Didier RETOUR

Université Pierre-Mendès-France, Grenoble II

\section{MOTS CLÉS}

\section{PME - Gestion des compétences - Entreprise en croissance}

\begin{abstract}
RÉSUMÉ
La recherche sur les PME a jusqu'ici mis en lumière les enjeux ressources humaines de la croissance ainsi que l'existence de pratiques de gestion des compétences. Mais, de façon surprenante, ces deux dimensions n'ont pas été croisées jusqu'à
\end{abstract}

\section{LES AUTEURS}

ChRISTIAN DEFÉLIX est professeur de sciences de gestion et spécialiste en ressources humaines à I'Université Joseph-Fourier, Grenoble I. II est aussi professeur affilié à l'École supérieure des affaires de l'Université Pierre-Mendès-France. En plus d'être chercheur au Centre d'études et de recherches appliquées à la gestion (Unité mixte de recherches CNRS 5820), il est l'auteur de plusieurs articles et contributions sur l'évolution de la fonction ressources humaines et les systèmes de gestion des compétences. II a agi à titre de coordinateur de RH Grenoble 2003 lors du XIVe congrès de l'Association francophone de Gestion des ressources humaines et VIe Université de l'Association nationale des directeurs et cadres de la fonction Personnel $<$ www.rhgrenoble2003.org >. Adresse : CERAG, Université Joseph-Fourier, Grenoble I, B.P. 47, 38040 Grenoble Cédex 9, France. Téléphone : 04768257 53, télécopieur : 0476546068. Courriel : <christian.defelix@imag.fr>.

DIDIER RETOUR est professeur de sciences de gestion et spécialiste en ressources humaines à l'École supérieure des affaires de Grenoble de l'Université Pierre-Mendès-France. Tout en étant chercheur au centre d'études et de recherches appliquées à la gestion (CERAG UMR CNRS 5820), il dirige l'École supérieure des affaires de Grenoble (Université-PierreMendès-France). II a présidé le comité d'organisation de RH Congrès 2003 et rédigé de nombreux articles en GRH et en management, notamment sur la gestion provisionnelle des emplois et des compétences, sur la gestion des compétences et sur la délégation. Adresse : Directeur de l'École supérieure des affaires Université Pierre-Mendès-France, Grenoble II, B.P. 47, 38040 Grenoble Cédex 9, France. Téléphone : 04768256 19, télécopieur : 0476825999. Courriel : <didier.retour@esa.upmf-grenoble.fr>. 
présent : que sait-on de l'apparition et du développement de la gestion des compétences dans les PME en croissance? Le présent article propose un cadre d'analyse et l'applique à une étude de cas longitudinale et approfondie, celle de l'entreprise Microtek.

\begin{abstract}
Management research enlightened the human resource issues of business growth and developed many observations about skill-based systems. Therefore, those two issues were never studied together, with a same point of view: what can we say about the management of skills in growing small business? This article offers an analysis grid for a longitudinal case study, the one of Microtek.
\end{abstract}

\title{
RESUMEN
}

Hasta el momento la investigacion sobre las pequenas y medianas empresas enfocado sobre los desafios de los recursos humanos para el desarollo de la empresa. y la existencia de practicas de gestion de las habilidades. Pero, sorprendentemente, estas dos dimensiones no han sido estudiadas de manera conjunta: que sabemos de la aparicion y del desarollo de la gestion de las habilidades de una pequena y mediana en crecimiento ? Esta articulo propone un marco de analisis y lo aplica al estudio amplio y profundo del caso de la empresa «Microtek».

\section{ZUSAMMENFASSUNG}

Die Forschung über die KMU hat bis jetzt den Einsatz der personellen Ressourcen in Wachstumsphasen sowie die Existenz von Managementkompetenz in den Vordergrund gesetzt. Erstaunlicherweise wurden diese zwei Dimensionen bis jetzt noch nicht quer verglichen: Was weiss man von der Entwicklung von Managementkompetenzen in stark wachsenden KMU ? Der vorliegende Artikel schlägt einen Analyserahmen vor und verwendet diesen bei einer ausführlichen Fallstudie Unternehmung «Microtek»-an.

\section{Introduction}

Longtemps considérée comme un type d'organisation relevant d'une gestion avant tout pragmatique et informelle, la petite et moyenne entreprise (PME) fait l'objet d'un regard plus nuancé et approfondi depuis quelques années (Heneman, Tansky et Camp, 2000). Les recherches en gestion des ressources humaines (GRH) ont en particulier bien fait apparaître l'existence de besoins en la matière (d'Amboise et Garand, 1995) et de véritables préoccupations de GRH dans ce type d'organisation (Bayad et Nebenhaus, 1996). Même si les modalités de prise en charge de la fonction diffèrent selon la taille (Mahé de Boislandelle, 1998a, p. 25-64), les questions de recrutement (Garand et Fabi, 1994), mais aussi de motivation et de formation (Paradas, 1998), sont au cœur des préoccupations des dirigeants de PME. 
Or, si bien des PME sont amenées à rester petites ou moyennes (Girard, 1997), l'expérience de la croissance reste fréquente pour bien des organisations de cette catégorie, ne serait-ce que dans le passage de la «très petite » à la "petite », voire à la «moyenne » organisation. La littérature organisationnelle a assez largement étudié les étapes de structuration franchies dans ce contexte de croissance, tandis que les chercheurs en GRH ont bien éclairé au cours des 10 dernières années l'évolution globale des pratiques de GRH en lien avec la hausse des effectifs et de l'activité. Par ailleurs, les observateurs commencent à élargir aux PME le repérage et l'analyse des systèmes de gestion des compétences, initialement étudiés dans les grandes organisations seulement. En effet, avec la montée en importance des choix à opérer en matière de recrutement, d'emploi et de formation, on est fondé à parler d'une réelle gestion des compétences pour les PME, au sens de pratiques visant à acquérir, stimuler et réguler les compétences des salariés plutôt que les seules qualifications ou les seuls effectifs.

Curieusement, ces deux dimensions de recherche n'ont pas été prises en compte simultanément jusqu'ici : les travaux sur la croissance des PME parlent peu ou pas de gestion des compétences et les analyses portant sur la gestion des compétences n'incorporent que très rarement la problématique de croissance des petites et moyennes entreprises. Que peut-on dire alors de la gestion des compétences dans les PME en croissance ? Quelles sont les conditions d'apparition et de développement d'une telle gestion dans ce contexte particulier? Et notamment, la formalisation qui accompagne inévitablement toute croissance organisationnelle oriente-t-elle d'une manière particulière le management des compétences des salariés ? Pour répondre à ces questions, nous proposons ici un cadre d'analyse (section 1); puis l'étude du cas d'une PME de haute technologie (section 2) et son interprétation (section 3) donneront lieu à une discussion théorique sur le développement de la gestion des compétences dans les PME en expansion.

\section{Problématique et cadre d'analyse: étapes de croissance et gestion des compétences, deux dimensions à croiser pour la recherche en PME}

Le développement des organisations, au sens de la croissance de leur taille et de leur volume d'activité, est depuis longtemps un sujet d'intérêt pour les chercheurs. Cet intérêt a été renforcé au cours des 15 dernières années par les avancées de la recherche en GRH sur les PME. Mais dans toutes ces études, la gestion des compétences n'est pas réellement prise en compte (1), tandis que de leur côté, les recherches sur les systèmes de gestion des compétences ne s'intéressent pas à la problématique d'une GRH de croissance (2). Or, bien des enjeux théoriques et pratiques se tiennent derrière la prise en compte simultanée de ces deux objets de recherche, que nous proposons d'appréhender par une étude longitudinale (3). 


\subsection{La GRH des PME en croissance... ou la compétence oubliée ?}

La problématique de la croissance des organisations a nourri de nombreuses recherches dont les synthèses nous proposent des modélisations très intéressantes du développement des organisations à travers le temps. Il y a près de 30 ans, Greiner (1977) avançait que dans le processus de développement d'une entreprise alternaient phases d'évolution et de révolution et qu'une organisation «mûre » passait fréquemment par des crises de leadership, d'autonomie, de contrôle et de bureaucratie. Dans cette célèbre étude organisationnelle, la gestion des ressources humaines en tant que telle n'était abordée qu'indirectement, à travers des thèmes génériques de motivation ou de communication et le concept de compétence, lui, était absent. Dans une perspective voisine, Churchill et Lewis (1983) ont proposé une modélisation du processus de croissance en cinq étapes : l'apparition, la survie, le succès, le décollage et la maturité des ressources. Parmi les dimensions clés repérées par les auteurs, les ressources en personnel et la qualité des collaborateurs sont considérées comme déterminantes, sans toutefois que les compétences des salariés soient explicitement citées. Le constat est le même pour une contribution plus récente, celle de Hanks et al. (1993), basée sur un échantillon de 126 entreprises de haute technologie: si la fonction Personnel y est explicitement repérée dans l'étape 4 et si la formalisation des politiques ressources humaines est observée, la problématique des compétences ne figure pas.

Toutes ces recherches relèvent en fait du champ du management stratégique ou de l'entrepreneuriat, peu familiers des questions de gestion des compétences individuelles ${ }^{1}$. La synthèse entre la GRH et le management des PME reste à construire, comme le remarquaient récemment Katz et al. (2000). Néanmoins, tout un courant francophone de recherche en gestion des ressources humaines dans les PME a, depuis plus de 10 ans, apporté sa contribution à cette synthèse. Ainsi, les repérages effectués par Kalika (1988) et Mahé de Boislandelle (1998a) ont établi que l'atteinte d'un effectif entre 150 et 200 salariés représente pour beaucoup d'entreprises un tournant dans la prise en charge formelle de la fonction : la présence d'un responsable de personnel assorti d'un service plus ou moins étoffé est constatée et la conscience de l'importance des choix à opérer en matière de recrutement, d'emploi et de formation augmente sensiblement. Les « difficultés de compétences » sont même citées comme l'un des principaux problèmes qui se posent alors dans le domaine de la GRH (Mahé de Boislandelle 1998a, p. 52), ce qui montre, si besoin était, l'importance d'étudier de plus près la gestion des compétences dans ce contexte de croissance.

1. Dans le domaine du management stratégique, la gestion des compétences renvoie en effet aux compétences organisationnelles, non aux compétences individuelles qu'étudient les chercheurs en GRH. Même s'il s'agit du même mot, les objets sont très différents, comme l'ont noté Aubret, Gilbert et Pigeyre (2002, p. 73-76). 


\subsection{La gestion des compétences... ou les PME en croissance négligées?}

Si l'on se tourne à présent vers la littérature de GRH consacrée à la gestion des compétences individuelles, on ne trouve pas pour autant d'éclairages révélateurs sur la problématique des PME en croissance. Une première raison en est que les exemples et références utilisés par cette littérature relèvent avant tout du monde des grandes organisations : ainsi, dans leur panorama historique, Aubret, Gilbert et Pigeyre (2002, p. 15-20) citent IBM, Sollac Dunkerque ou les grandes banques. Et de fait, en matière de gestion des compétences, ce sont les grandes entreprises qui sont traditionnellement montrées en exemple, telles Usinor, EDF-GDF ou Hewlett-Packard. Ce n'est que depuis peu qu'on ose parler de gestion des compétences à propos des petites et moyennes entreprises (PME) : ainsi, en France, le trophée décerné par le cabinet CEGOS et la revue française Enjeux distinguait, fin 2001, deux PME parmi les trois entreprises lauréates. Bien entendu, les PME n'ont pas attendu qu' on parle d'elles pour entreprendre et développer de réelles démarches instrumentées de gestion des compétences. Mais celles-ci sont à l'heure actuelle insuffisamment identifiées et surtout implicitement dévalorisées, notamment parce qu'il est fréquent de leur projeter le référentiel des grandes entreprises : le risque d'un tel biais a été clairement mis en évidence (Nebenhaus et Sarnin, 1993 ; Bayad et al., 1995).

La seconde raison de l'absence de prise en compte réelle de la problématique de PME en croissance dans les travaux consacrés à la gestion des compétences tient à ce qu'il y a encore très peu de travaux de recherche empirique sur la gestion des compétences au sein des petites et moyennes organisations : dès lors, les chercheurs qui s'y intéressent en sont encore à une optique exploratoire, visant à lever le voile sur les pratiques de terrain. Ainsi Scouarnec (1999), en se basant sur l'étude d'une série d'accords conclus au sein d'entreprises moyennes, discerne le recours à une gestion des compétences plutôt contrainte dans les entreprises moyennes, basée sur le requis et relevant d'une démarche instrumentale. De leur côté et cette fois, en se basant sur une étude de cas au sein d'un établissement bancaire français de taille moyenne, Leray et Joyeau (1999) relèvent non seulement l'existence d'une gestion prévisionnelle des emplois et des compétences, mais aussi un processus d'aller-retour entre cette pratique et l'évolution de la structure organisationnelle. Avec une autre méthodologie, en l'occurrence une série de cas exploratoires, Defélix, Dubois et Retour (1999) ont observé l'existence de dispositifs «maison » pour gérer les compétences, reliés de façon variable avec la stratégie et présentant des degrés de formalisation très inégaux. Quant à Géniaux et Mira Bonnardel (2001), elles ont confirmé récemment de manière indirecte le caractère stratégique d'une gestion des compétences en PME, en mettant en lumière la prégnance de la gestion des savoirs même pour la petite entreprise. 
Les travaux portant sur la gestion des compétences en PME commencent donc à se développer, mais pour l'heure, ils restent focalisés sur des problématiques larges et non sur le rôle que peut jouer un dispositif de gestion des compétences dans une phase de croissance.

\subsection{PME en croissance et gestion des compétences : pour une analyse longitudinale}

Modélisations de PME en croissance d'un côté, recherches encore exploratoires sur la gestion des compétences en PME de l'autre : on le voit, ces deux objets sont encore excessivement cloisonnés. Cette situation est d'autant plus paradoxale que les spécialistes patentés de la PME ont clairement mis en évidence la problématique d'adaptation des ressources humaines aux impératifs stratégiques (Naro, 1990). Il paraît donc utile et même urgent de s'interroger sur l'existence, la configuration et l'impact des pratiques de gestion des compétences dans un contexte de croissance. Si les pratiques de gestion des compétences existent dans les PME, prennent-elles des formes particulières dans les PME en croissance ? L'évolution des effectifs et de l'activité joue-t-elle un rôle stimulant pour la gestion des compétences ou, au contraire, relègue-t-elle cette gestion à un second plan ? L'inévitable formalisation qui accompagne toute croissance favorise-t-elle l'instrumentation et si oui, laquelle? Oriente-t-elle la gestion des compétences vers une optique de formalisation de ce qui est requis sur les emplois, ou cible-t-elle prioritairement les compétences détenues par les individus?

De telles questions nous orientent vers une perspective dépassant le seul caractère instrumental de la gestion et s'ouvrant à la dimension temporelle et évolutive des pratiques. C'est dans cette perspective que nous retenons ici le cadre théorique de l'analyse contextualiste (Pettigrew, 1985), que plusieurs auteurs appliquent d'ores et déjà à l'étude des changements dans les systèmes de gestion des ressources humaines (Brouwers et al., 1997; Pichault et Nizet, 2000). Cette théorisation souligne en effet «la nécessité de dégager les interconnexions temporelles. Il s'agit d'une tentative de capter la réalité "en vol". Le chercheur centré sur les processus aura comme ambition fondamentale de comprendre la séquence et le flux des événements au cours du temps. L'histoire est à cet égard cruciale » (Pettigrew, 1985, p. 8 ; Brouwers et al., 1997). Cette approche considère les cas de changement organisationnel à l'aune de trois variables :

1. Le contenu du changement. Il s'agit du domaine concerné par le changement, auquel le chercheur a décidé de s'intéresser. Dans notre cas, il s'agit des pratiques et des outils par lesquels sont acquises, stimulées et régulées les compétences individuelles : définitions de fonction stipulant les compétences requises, support d'appréciation, dispositif de formation, etc. 
2. Le contexte. Il s'agit aussi bien de l'environnement de l'organisation (social, politique, économique...) que des caractéristiques internes : sa structure, sa culture, son style de management. Appliquée à notre objet de recherche, cette notion de contexte renvoie à l'environnement externe et son impératif de flexibilité, mais aussi aux caractéristiques internes de la PME (organisation du travail, population, culture...).

3. Les processus, c'est-à-dire les initiatives des acteurs et les rapports de pouvoir qui se développent entre eux. Sur le thème de la gestion des compétences, ces acteurs peuvent être, comme pour toute situation de GRH, le dirigeant, le responsable des ressources humaines, les cadres, les syndicats, etc. Comme le remarquent Pichault et Nizet (2000, p. 32), «d'un point de vue contextualiste, on considère [...] que les jeux d'acteurs sont contraints, au moins en partie, par les contextes, mais en même temps qu'ils les construisent [...] et ainsi les transforment ».

Ce cadre théorique nous a conduits à une collecte de données longitudinale, auprès d'une entreprise du secteur de la microélectronique, Microtek ${ }^{2}$. D'une part, 22 entretiens semi-directifs ont été réalisés en 2001 et 2002 auprès d'un échantillon contrasté du personnel : ces entretiens ont permis de rencontrer le directeur général et cofondateur, le DRH et le responsable formation, cinq ingénieurs et membres de l'encadrement, sept techniciens, six opérateurs et un délégué syndical. D'autre part, nous avons pu accéder à des documents internes relevant d'époques différentes, tandis que plusieurs réunions de restitution avec la direction des ressources humaines de cette organisation ont permis de suivre, en 2001, 2002 et 2003, la croissance accélérée de cette entreprise et les évolutions internes que celle-ci induisait. L'observation a été complétée par plusieurs visites de sites ainsi que le suivi d'étudiants spécialisés en GRH effectuant leur stage au sein de cette société.

\section{Le cas Microtek : la structuration progressive d'un système de gestion des compétences dans un contexte de croissance accélérée}

Microtek, comme bien d'autres entreprises de haute technologie (Jolly et Roche, 1999), cherche sans cesse à s'adapter aux réalités de son secteur pour survivre et poursuivre sa croissance. Mais alors que dans les grandes entreprises la gestion des compétences est plutôt censée stimuler la flexibilité et l'innovation du personnel, elle est mise en œuvre chez Microtek pour jouer un rôle inattendu et paradoxal de structuration et de rationalisation. Pour le comprendre, voyons d'abord quelle est

2. Il s'agit d'un nom fictif pour une entreprise réelle. Les localisations géographiques indiquées dans la suite du texte ont également été maquillées. 
l'histoire de cette jeune entreprise (1), avant d'examiner son système de gestion des compétences (2), puis de saisir la diversité des représentations du personnel face à ce système (3).

\subsection{Microtek ou l'itinéraire d'une entreprise gâtée}

Microtek est une jeune entreprise du secteur de la microélectronique, dont l'activité consiste à traiter des plaques de silicium afin de les rendre plus performantes. Cette «jeune pousse » voit le jour en 1992, à partir d'un brevet développé dans un grand laboratoire d'électronique. Ses dirigeants - l'un est un chercheur de haut niveau, l'autre un ingénieur - développent en 1994 et 1995 un nouveau procédé, le «Microtek Plus », technologie exclusive physicochimique dont le principe est de découper à l'infini de fines tranches de silicium et de les appliquer sur un support mécanique. Ce procédé induit des propriétés électroniques supérieures : les performances des semi-conducteurs sont améliorées de $20 \%$ et la consommation d'énergie est réduite de $30 \%$ par rapport au silicium brut, le tout pour un surcoût limité à $10 \%$.

C'est en 1996 que Microtek passe le cap des 50 employés. La direction décide alors d'intégrer un spécialiste des ressources humaines plutôt que de recourir à un cabinet extérieur. Romain Levesque est recruté comme directeur des ressources humaines (DRH) en juillet 1996, après un parcours dans une filiale semi-conducteurs d'un grand groupe et une expérience de DRH à temps partagé. Le même souci d'anticipation est illustré en 1996 par le recrutement d'un directeur financier de stature internationale, chargé de préparer une future introduction en Bourse.

La direction de Microtek veut alors construire une usine spécifique, mais se heurte à la difficulté de trouver l'argent nécessaire, à une époque où ni l'université ni les banquiers ne croient encore véritablement dans le potentiel du nouveau procédé. L'ouverture du capital à un producteur japonais de silicium permet aux dirigeants de trouver néanmoins les ressources financières indispensables à la construction d'un bâtiment industriel.

La «factory » est ainsi ouverte en juillet 1998. Ensuite, 1999 est une année charnière pour Microtek: outre l'inauguration du site de Palaiseau, la société acquiert la certification ISO 9001 en janvier et entre au Second Marché de la Bourse de Paris en février. L'argent collecté en Bourse permet à l'entreprise, par un effet de levier, de continuer à investir. La niche de Microtek commence peu à peu à être rentable: en 2000, alors que le marché des plaques de silicium sur isolant représente 50 millions de dollars, Microtek en détient $80 \%$. Une augmentation de capital d'un montant de 92 millions d'euros est réalisée en juin 2000. En 2001, le résultat d'exploitation devient positif. 
En septembre 2001, Microtek emploie 223 salariés : 143 hommes et 80 femmes, avec une moyenne d'âge de 30 ans et une structure de qualification presque équilibrée en trois tiers (37,6\% d'opérateurs, 33,2\% d'employés et de techniciens, et $29,2 \%$ de cadres). La feuille de route du DRH consiste à assurer la croissance de l'effectif (120 recrutements dans l'année), mais aussi à renforcer le management et à répondre aux aspirations des techniciens en termes d'évolution professionnelle. Le début de l'année 2002 confirme ces chiffres optimistes : la direction annonce un chiffre d'affaires consolidé pour le deuxième trimestre de l'exercice à 20,8 millions d'euros, en croissance de $174 \%$ par rapport au même trimestre 2000-2001. Dans un environnement économique pourtant tendu, notamment sur le marché des semiconducteurs, la demande pour la technologie Microtek Plus n'a pas faibli et l'ouverture d'un deuxième site de production, « Palaiseau II », a eu lieu en septembre 2002.

Dans ce contexte de croissance accélérée, la direction de l'entreprise met progressivement en place une gestion des compétences. L'analyse des documents et les entretiens que nous avons menés font apparaître une structuration dans cette gestion, qui n'est pas sans provoquer des réticences en interne.

\subsection{Gérer les compétences chez Microtek : une préoccupation initiale, un système qui se formalise par palier}

Selon le DRH, la gestion des compétences chez Microtek a toujours été une préoccupation transversale, qui «impacte tout le processus RH. J'ai l'impression qu'on traite le recrutement, l'intégration, la formation, la fidélisation... sous un axe compétence ». Cette préoccupation a en fait donné lieu à une formalisation par palier, d'abord en 1998 et surtout en 2001. Voyons-en les caractéristiques à travers les principaux éléments de la gestion des ressources humaines: le recrutement, la classification, l'évaluation et la formation.

Le recrutement vise plus que les compétences nécessaires à l'instant $t$. Le principe de base, non écrit mais commun aux hiérarchiques et à la direction, est dès l'origine de recruter avec une marge de deux années. «L'expression consacrée chez nous est: il faut qu'il en ait sous la pédale!», dit le DRH. Le comité de direction se pose au moins une fois par an la question des besoins par rapport au plan d'affaires. Au démarrage de l'entreprise, le repérage des compétences se fait sur un mode informel: un employé engagé chez Microtek en 1996 décrit être «rentré par connaissance, recruté directement par monsieur Collet, je suis allé dans son bureau et j'ai négocié avec lui mon salaire ». Des définitions de poste n'ont été formalisées qu'à partir de 1998, à la faveur de la démarche qualité, avant d'être mises en cohérence en 2001 avec les termes de l'entretien annuel. On y retrouve ainsi aujourd'hui les «compétences techniques requises» (comme la maîtrise des procédés de fabrication, la connaissance des matériaux...), ainsi que les «compétences personnelles nécessaires» qui articulent les trois familles 
d'aptitudes (concrétiser, communiquer et anticiper). Des profils de poste (une trentaine), décrivant en détail les capacités personnelles requises, sont de plus utilisés pour le recrutement. Des profils de compétences, certes moins développés et qui demeurent assez génériques, sont depuis peu accessibles sur Internet.

Le salarié, une fois intégré, est positionné en référence à la classification de l'Union des industries métallurgiques et minières (UIMM). L'entreprise y surajoute néanmoins depuis 1998 un processus de reconnaissance et de valorisation des compétences détenues dans le cadre d'une démarche d'habilitation progressive des opérateurs : le coefficient de rémunération augmente selon que l'on franchit les niveaux, ce qui, dans une entreprise où les salaires des opérateurs sont réduits, se veut une incitation à la formation. Le niveau 0 est celui des deux premiers mois de l'opérateur et sert de période d'accueil, au cours de laquelle l'intéressé ne peut travailler seul en salle blanche. Le niveau 1, qu'un opérateur occupe depuis son intégration jusqu'à la fin de ses six premiers mois environ et qui correspond au coefficient 170, dénote une capacité de l'opérateur à travailler seul, mais sous la direction du superviseur. Le niveau 2, que l'on peut briguer ensuite et qui s'accompagne du coefficient 190, décrit un opérateur capable de gérer complètement son poste et de participer à la formation des nouveaux. Le niveau 3 est en train de se mettre en place début 2002 : selon le DRH, «c'est le tutorat, c'est-à-dire la capacité à transmettre son savoir-faire ». Un processus semblable est mis en place pour les techniciens au cours de l'année 2002.

L'évaluation annuelle ne portait pas, à l'origine de l'entreprise, sur les compétences. Selon le DRH, «c'était un entretien entre le DG et chacun pour déterminer le pourcentage d'augmentation de chacun ». Puis un premier support d'évaluation est défini, peu de temps après son arrivée, par le DRH, support qui différencie les opérateurs des techniciens et cadres: le support d'évaluation des opérateurs ne laisse que quelques lignes relatives aux «compétences du salarié », mais on y retrouve pêle-mêle des « techniques sur le poste de travail», la « qualité et le résultat obtenu» et «l'organisation sur le poste». Le support d'évaluation des techniciens et cadres, en revanche, prévoit une page pour faire apparaître les «principales compétences techniques nécessaires » ainsi que les «principales compétences personnelles nécessaires ». Les deux supports ont en commun de ne pas inviter explicitement à formuler des objectifs de progression sur ces différentes compétences.

Or, une consultation menée en interne au printemps 2001 fait remonter plusieurs types d'insatisfaction des managers face à ces documents : selon eux, le libellé des compétences personnelles est variablement interprété, l'appréciation qui en est faite est floue, voire complaisante pour ne pas faire de vagues. Un nouveau support, en l'occurrence unique, est défini à la suite de cette consultation. Il se compose de six pages: une pour l'appréciation de la réalisation des objectifs au cours de la période précédente, trois pour l'évaluation des compétences (selon une 
distinction clarifiée: connaissances techniques requises, aptitudes personnelles, compétences professionnelles induites), une pour la détermination des objectifs de la période à venir et une pour les souhaits d'évolution et la synthèse de l'entretien. Le dispositif prévoit désormais de séparer le moment de l'entretien (mai) et celui de la détermination des augmentations annuelles (septembre) «pour ne pas polluer l'entretien», dixit le DRH.

La définition retenue de la compétence chez Microtek n'a d'ailleurs été formalisée que dans le courant de l'année 2001. Une compétence professionnelle y est entendue comme « un savoir-faire validé, mis en œuvre systématiquement en situation professionnelle et de façon autonome »; elle procède à la fois de connaissances techniques et d'aptitudes personnelles. Celles-ci sont référencées dans un document distinguant les aptitudes à concrétiser (p. ex., la capacité à planifier), les aptitudes à communiquer (informer, écouter, gérer les conflits...) et les aptitudes à anticiper (connaissance de l'environnement, réactivité...).

Des possibilités de formations diplômantes sont organisées pour les opérateurs désirant devenir techniciens ou les techniciens souhaitant passer ingénieurs. Le DRH a mis en place un système de chartes d'évolution, par lesquelles une formation diplômante est financée par l'entreprise et effectuée en partie sur le temps de travail. Environ 10 chartes sont signées par an. Le souci du DRH est qu'avec ce système les personnes qui souhaitent accéder à un poste donné en aient effectivement la qualification: «Le monde est ainsi fait: si demain l'entreprise ferme ses portes, les ingés auront du mal à trouver un emploi. Et les gens qui deviennent ingénieur maison par l'expérience, il leur manque un développement de connaissances théoriques, pilier de leurs compétences professionnelles. [...] Cela étant dit, il y a chaque année des gens qui sont promus sans diplôme, à mon corps défendant. »

Côté développement des compétences, $10 \%$ de la masse salariale sont aujourd'hui consacrés à la formation, dont les formations au poste (6\% sinon). Les objectifs du plan de formation sont déterminés à partir du comité de direction, des entretiens annuels et du recensement des besoins auprès des managers. Il n'existe pas de gestion prévisionnelle des ressources humaines en tant que telle, ni de revue de personnel, sinon de manière informelle. Le «bouclage» entre les orientations stratégiques et les besoins s'exprime en comité de direction une fois par an.

En 2001, le DRH entreprend un travail de formalisation d'un cursus de formation pour ses managers. Un consultant extérieur vient animer un groupe de travail composé de hiérarchiques et chargé de faire émerger les thèmes prioritaires devant être traités dans ce cursus. Il en est notamment ressorti la nécessité pour le manager Microtek d'être «un homme d'équilibre », dans la mesure où il s'agit de gérer plusieurs paradoxes : «être leader» et «faire adhérer et partager », «clarifier et exiger » mais aussi «faire progresser en autonomie», « organiser et structurer» 
tout en faisant en sorte « d'intégrer le changement »... six à huit jours de formation sont prévus dans cette optique, articulant les «fondamentaux » (organisation de l'entreprise, législation sociale...), le «quotidien» (le recrutement, la conduite d'entretien...) et la « complexité » (la conduite de changement...).

\subsection{Les représentations du personnel relativement au système : une légitimité reconnue, mais des modalités contestées}

Les entretiens menés auprès des opérateurs, des techniciens et des cadres de Microtek font apparaître, au sujet de ce système qui s'est structuré en 1998 puis en 2001, des positions mitigées : la notion même de gestion des compétences reste floue; pour autant, la préoccupation de gérer les compétences est reconnue comme légitime et surtout, les modalités que prend cette préoccupation sont souvent contestées.

Tout d'abord, la capacité à expliquer ce que recouvre cette notion de gestion des compétences est variable au sein du personnel, allant de la définition complète à l'incertitude : «Le développement des compétences, donner des compétences aux gens » (ingénieur), «Mettre en application ce que l'on connaît le mieux et faire l'effort pour apprendre » (assistante), «Pas très clair pour moi » (ingénieur), «C'est relativement flou » (opérateur), « Je rattacherais ça à l'expérience, les études, ce que la personne sait faire et ne pas faire, pour mieux la placer, qu'elle soit au bon endroit » (technicien).

Néanmoins, pour l'ensemble des personnes rencontrées, il s'agit de quelque chose d'important, que l'entreprise pratique effectivement ou à l'inverse qui lui manque. Les salariés de l'entreprise, même s'ils n'ont pas tous une vue claire et complète de ce que signifie gérer les compétences, y accordent de l'importance et sentent que peu à peu cette démarche devient celle de toute l'entreprise : « La gestion des compétences, c'est la gestion des ressources humaines » (ingénieur), «Avec l'augmentation du nombre de personnes, c'est vraiment défini comme un axe de progrès nécessaire » (ingénieur), «Je sais pas comment ça se passe dans les autres services, mais j'ai l'impression qu'on ne gère pas les compétences. On tient pas compte du potentiel des gens » (technicien).

Les représentations des salariés sur la question se révèlent, en fait, diverses selon la catégorie. Ainsi, les opérateurs associent spontanément la gestion des compétences au parcours d'habilitation progressive qu'ils suivent durant environ trois ans à partir de leur prise de poste. Les techniciens, de leur côté, expriment majoritairement une représentation négative de la gestion des compétences, au motif qu'ils ne se sentent pas reconnus et gérés dans leurs compétences. Les ingénieurs et cadres, enfin, relient la gestion des compétences à leurs pratiques et difficultés quotidiennes dans le management de leur service. 
Certaines caractéristiques du système de gestion des compétences concentrent l'attention des personnes interrogées. L'entretien annuel est ainsi reconnu comme à la fois nécessaire et mal positionné : «Je ne pense pas que l'entretien annuel m'apporte grand-chose. [Certes] on y parle de relations humaines, d'objectifs, de compétences souhaitées dans le poste. Par contre, rien sur l'évolution personnelle souhaitée, idem sur les souhaits de rémunération» (ingénieur).

C'est surtout les modalités de développement des compétences à travers une formation diplômante qui provoquent un sentiment d'amertume chez de nombreuses personnes : «Ils veulent me voir reprendre les études, ils sont prêts à faire une charte avec moi. [...] Moi, ce que j'espérais, c'est qu'avec un bac+4 et 10 ans d'expérience, ils me passeraient ingénieur, mais les ressources humaines ne veulent pas sans diplôme. J'ai la gorge qui se serre, je le veux mais je le peux pas. [...] Je trouve que c'est c... : ils ont une vision comme ça, "Si vous avez pas le diplôme vous avez pas le poste", je trouve qu'ils raisonnent comme des tambours, alors qu'on se dit entreprise jeune, à l'américaine, high-tech... Le poste d'ingénieur, ils savent très bien que je peux l'assumer, les compétences, je les ai, ils le savent. [...] Ils m'ouvrent une porte, mais ils me mettent des poids aux pieds » (technicien). "L'évolution est très limitée, aucun opérateur n'est technicien sauf un. Le passage obligé, c'est le retour aux études et c'est très difficile» (opérateur).

Au terme de la présentation du système de gestion des compétences au sein de cette moyenne entreprise en forte croissance et des représentations du personnel de Microtek en ce qui a trait à ce système, quelle interprétation et quelle discussion théorique peut-on mener?

\section{Interprétation : dans un contexte de croissance, un jeu d'acteurs dominant}

L'interprétation théorique sera conduite, comme nous l'avons annoncé, au moyen de l'analyse contextualiste qui s'appuie sur trois concepts clés et leurs interrelations : le contenu, en l'occurrence le système de gestion des compétences (1), puis les éléments de contexte (2) et, enfin, les processus, soit le jeu des acteurs (3).

\subsection{Le contenu : un système hybride pour gérer les compétences}

À l'analyse, le système de gestion des compétences dont Microtek s'est progressivement doté apparaît hybride à plusieurs égards.

En premier lieu, la définition retenue par l'entreprise de la compétence « un savoir-faire validé, mis en œuvre systématiquement en situation professionnelle et de façon autonome »- ne mentionne pas explicitement les savoirs et les attitudes (savoir-être). Pour autant, ce «savoir-faire » est décrit, nous l'avons vu, 
comme étant engendré par des connaissances et des aptitudes... Et d'ailleurs, l'entreprise insiste sur l'importance, pour les salariés qui veulent progresser, d'acquérir des connaissances (à travers le programme de formation diplômante) et sur le poids que revêt l'évaluation des compétences relationnelles dans l'entretien annuel. Ainsi, une confusion peut exister entre la définition mise en avant par l'entreprise et les trois dimensions génériques de la compétence généralement admises : savoir, savoir-faire, savoir-être (Durand, 2000).

Une deuxième source d'hybridation relève de l'orientation retenue pour la gestion des compétences: l'importance donnée aux définitions de fonction et le recours à la classification UIMM correspondent à une logique traditionnelle des compétences requises sur l'emploi, tandis que la pratique des habilitations progressives concrétise une logique d'évaluation et de rémunération des compétences détenues. L'accent est donc volontairement mis sur l'évaluation des compétences tant requises que détenues. Les salariés doivent comprendre que leur développement sera fonction de l'augmentation de leur capital compétence, d'autant plus que l'entreprise est appelée à mobiliser chaque jour davantage plus de savoir-faire spécialisés, compte tenu de ses évolutions technologiques et du développement de ses lignes de production. Cela peut aussi s'interpréter comme une difficulté à définir les objectifs que devra atteindre chaque salarié. En effet, si les objectifs stratégiques de l'entreprise semblent être clairement précisés, ce ne serait pas le cas pour les objectifs individuels qui, notamment, ne seraient pas aussi formalisés que dans d'autres entreprises pratiquant ce type de management. Il s'agirait davantage de «priorités » à respecter que de véritables objectifs, au sens habituel du terme. Le DRH confirme le sentiment de quelques salariés sur ce point en déclarant : "On sait très bien faire les plans stratégiques. Après, pour la déclinaison en objectifs individuels, on n'est pas bon. » Un ingénieur interviewé résume parfaitement le défi à relever pour l'entreprise en matière d'évaluation : "Les entretiens sont plus axés sur les compétences que sur les objectifs et la performance. Si on arrivait à articuler les deux, ce serait parfait. » Dès lors, le «modèle de GRH » pratiqué par Microtek est au confluent du modèle individualisant et du modèle objectivant (Pichault et Nizet, 2000). Du modèle individualisant, Microtek conserve en effet la stimulation individuelle des salariés et la contractualisation des priorités avec la hiérarchie immédiate. Du modèle objectivant, Microtek emprunte l'existence de règles et de cadrages impersonnels, à l'image de cette standardisation des qualifications qui se met en place (tel diplôme pour tel poste).

Une troisième source d'hybridation se rapporte au caractère formel ou informel du système de compétences :

- d'un côté, le degré de formalisation auquel parvient le système de gestion de compétences chez Microtek ne laisse pas d'étonner. Certes, il subsiste dans l'ancienne start-up des pratiques informelles qui témoignent du caractère hybride déjà souligné. L’entreprise, par exemple, ne mobilise

Revue internationale P.M.E., vol. 16, $\mathrm{n}^{\text {os }} 3-4,2003$ 
pas d'outils très simples du type « organigrammes de remplacement » qui pourraient faciliter la tâche des dirigeants dans leur préparation de l'avenir et qui ne demandent pas trop d'efforts pour leur mise en œuvre. La société pratique une revue du personnel informelle et suit uniquement quelques personnes clairement reconnues comme «haut potentiel». De même, le DRH se refuse à toute gestion prévisionnelle des emplois et des compétences (GPEC), préférant surdimensionner le capital compétences à détenir au moment d'intégrer Microtek. En d'autres termes, on a affaire dans cette entreprise à une gestion anticipée des compétences (GAC) plutôt qu'à une GPEC au sens traditionnel du terme. Cela peut avoir du sens dans une entreprise qui évolue avec encore beaucoup d'incertitudes et peu de visibilité quant à ses besoins. Il s'agit alors de compter sur des hauts potentiels capables d'assurer des rôles multiples pendant les phases de croissance de l'entreprise (Heneman, Tansky et Camp, 2000);

- d'un autre côté, d'autres modalités du système de gestion des compétences vont pourtant dans le sens d'une très grande formalisation : pour le support d'évaluation, six pages sont à compléter dans une entreprise qui est encore de taille moyenne, c'est beaucoup surtout dans un contexte de croissance et d'urgence productive. La jeune société est-elle obligée de collecter autant d'informations dans le cadre du processus d'appréciation du personnel ? Doit-on l'interpréter comme une difficulté croissante à suivre finement ses salariés, mobilisés sur plusieurs projets à la fois ou travaillant désormais dans des conditions qui n'autorisent plus un suivi rapproché au quotidien? Ou s'agit-il d'une volonté de formaliser et de disposer de beaucoup de données sur ses salariés afin de mieux les manager dans une gestion globale des compétences ? Cette dernière hypothèse apparaît la plus crédible, mais la jeune société doit avoir à l'esprit les contraintes d'une telle collecte et ne pas transformer cette ambition en «usine à gaz » contre-productive.

La formalisation se double d'une volonté de standardisation étonnante pour une PME. En effet, s'il est possible grâce au nouveau dispositif de progresser, c'est avec une condition très claire de charte et de formation diplômante. Le système risque de rencontrer vite ses limites en ne permettant pas une stimulation durable du personnel. Seuls les salariés acceptant de participer à la démarche privilégiée par la direction des ressources humaines y trouveront leur compte. C'est un choix qui doit être parfaitement assumé par les dirigeants de l'entreprise et les salariés.

Les analyses et les interprétations données dans les paragraphes précédents aboutissent à la double conclusion suivante : au sein de Microtek, la gestion des compétences se formalise de plus en plus, tout en ne pouvant pas être considérée comme étant arrivée à un système pleinement cohérent et complet. Nous serions 
plutôt enclins à parler de grappes (bundles) de gestion des compétences que d'une véritable gestion des compétences totalement intégrée et pleinement finalisée. Cette situation reflète aussi quelque part l'influence du contexte.

\subsection{Le contexte : l'effet taille... et les autres facteurs de l'environnement interne et externe}

Les marques d'un phénomène de formalisation et de standardisation croissantes de la gestion des compétences observées chez Microtek s'expliquent par plusieurs facteurs contextuels.

Le premier qui vient à l'esprit est certainement la contingence de la taille. En effet, la croissance des effectifs impose de mettre en place entre la direction et l'exécution un ou plusieurs niveaux intermédiaires de responsabilités, auxquels différentes fonctions sont attribuées et qui disposent d'objectifs, de moyens et d'autorité. À ce stade surgissent les premières difficultés de formalisation des rôles et des relations qui tendent à se dépersonnaliser du fait de l'augmentation du nombre d'individus. La coordination n'est plus un phénomène spontané. La taille induit une plus grande division du travail et la mise en place de procédures standardisées, afin d'assurer le traitement d'un volume de plus en plus important d'informations. La spirale bureaucratique (STRATEGOR, 1985) amorcée ne semble pas prête de prendre fin : la croissance du chiffre d'affaires - $100 \%$ pour le troisième trimestre de l'exercice 2001-2002 ; + $126 \%$ en cumul sur les neuf premiers mois de l'exercice, en pleine période de morosité de l'industrie des semi-conducteurs - donne tout son crédit à l'hypothèse, envisagée par la direction, d'un doublement des effectifs dans les prochaines années.

Néanmoins, l'effet taille ne rend pas pleinement compte du processus de formalisation et de structuration de la gestion des compétences chez Microtek. D'autres éléments sont indispensables à la compréhension; certains relèvent de l'environnement interne, d'autres de l'environnement externe :

- la formalisation est accentuée par la nature même des activités de Microtek, caractérisées par la croissance de la production ces dernières années et dont on sait par essence qu'elle induit des actions régulières, répétitives, traçables et formalisées ; ainsi, la dynamique d'industrialisation liée au nombre croissant de lignes de production conduit naturellement à des études statistiques plus élaborées et à la nécessité de documenter davantage les actions entreprises ;

- les formes organisationnelles adoptées, notamment depuis le démarrage opérationnel de la nouvelle unité de production. L'organisation du travail a été modifiée à cette occasion : quatre «zones» sont mises en 
place, chacune sous la responsabilité d'un responsable de production, correspondant à différentes parties du processus technique - polissage, support technique, etc.;

- il existe l'obligation de maîtriser chaque jour davantage la production pour répondre à la hausse de la demande des plaques de silicium traitées avec le processus technique établi par Microtek et les exigences de certification (ISO 9001) imposées par les clients et le marché. Dans ces conditions, les machines doivent être mobilisées au maximum. L'apprentissage est obligatoire et la formalisation croissante inévitable ! Mais tous les acteurs concernés n'ont pas la même sensibilité à l'égard de ce mouvement de fond, ce qui nous conduit à prendre en compte la troisième dimension de notre analyse : le processus.

\subsection{Le processus : une dynamique dans le jeu des acteurs}

Le recours à l'analyse du jeu d'acteurs est en effet essentiel pour la compréhension de l'évolution de la gestion des compétences dans cette PME. À cet égard, il convient d'avoir à l'esprit le choix des dirigeants d'orienter délibérément l'entreprise vers la croissance interne (lancement de nouvelles usines) et externe (lancement d'un réseau indépendant sous licence, développement de partenariats en vue d'une diversification des matériaux, etc.) tout en voulant recréer «le bouillon originel, maintenir le flou ». Au stade de développement de l'entreprise, peut-on vraiment faire ce pari? Il est en tout cas au cœur d'une tension dynamique entre deux groupes d'acteurs dans l'entreprise :

- d'une part, les salariés pionniers et la direction, toujours prudents quant à la formalisation;

- d'autre part, les ingénieurs et les cadres recrutés des grandes entreprises ainsi que la direction des ressources humaines, qui poussent à l'inverse à plus de mise par écrit et de standardisation.

Dans un premier temps, jusque vers 1998, le rôle de ces ingénieurs et de la DRH naissante est de fournir un léger contrepoids au bouillonnement créatif légitime de la PME high-tech. De la start-up, l'entreprise conserve encore un esprit pionnier et bouillonnant par certains côtés. C'est une entreprise dynamique avec du personnel jeune. Un ingénieur déclare : «C'est hyperdynamique avec une réactivité impressionnante. Par contre, on manque de formalisme. Nous sommes trop habitués à gérer l'urgence. On s'épuise un peu. » L'expression est très directe entre les salariés, quel que soit leur statut. On n'hésite pas à indiquer ce que l'on ne sait pas faire. C'est une culture de l'humilité. Personne ne se met en avant. Les salariés ont un esprit critique en sachant se remettre en cause si nécessaire. Ils n'hésitent pas à discuter, nous l'avons vu, directement avec le directeur général, si nécessaire.

Revue internationale P.M.E., vol. 16, nos 3-4, 2003 
À partir de 1998, le rapport tend à s'inverser et les acteurs de la formalisation prennent pour ainsi dire le pas. On ne comprendra pleinement les représentations de ces derniers qu'en référence au destin d'une autre PME de haute technologie, née dans le même laboratoire que Microtek. En effet, cette entreprise ferme ses portes en 1999: elle laisse alors sur le marché du travail des ingénieurs et des techniciens «maison», promus en interne sur la base de leurs compétences prouvées mais ne bénéficiant pas d'une qualification socialement reconnue. Et la DRH de cette autre PME n'était autre que l'épouse de Romain Levesque... Lequel en a peut-être tiré la conclusion que la standardisation des qualifications, même dans une PME, pouvait être une démarche prudente.

Le balancier est peut-être allé trop loin en la matière, du moins c'est ce que pense le directeur général qui déclare ainsi :

On est passé de l'âge de pierre, du bouillon mal géré mais efficace, à quelque chose de très structuré, trop je pense. Les gens se parlent moins [...] Je discutais hier avec une ingénieur, je me suis aperçu qu'elle faisait des manips qui se font ailleurs. Il faut créer des liens, de la synergie. On va ouvrir un portail, mettre des comptes-rendus dessus, que chacun apporte sa pierre. Il faut aujourd'hui recréer le b..., le bouillon. Il faut pas qu'il y ait le DRH d'un côté, la production de l'autre, on a aujourd'hui tendance à créer cette rupture. [...] Ce n'est pas en cloisonnant les zones que les gens vont se parler. Dans les nouveaux bâtiments, on réfléchit à l'implantation des bureaux : ne pas mettre l'informatique d'un côté, les autres ailleurs... Obliger la direction des techniques avancées à lancer des manips en production, maintenir le bouillon par l'organisation, en créer les conditions, maintenir du flou.

Il y a incontestablement un jeu subtil des acteurs où chacun, selon sa sensibilité, ses possibilités d'influence, ses zones d'incertitude, joue sur une dominante : informelle / formelle, individuelle / collective, compétence requise / détenue, etc., ce qui se traduit concrètement par le caractère hybride de la gestion des compétences présenté au paragraphe précédent.

\section{Discussion finale et conclusion}

Au total, plusieurs points de discussion peuvent être soulignés pour alimenter la recherche sur les PME en croissance. Le premier réside dans le caractère ambivalent de la gestion des compétences dans cette PME : dans la plupart des entreprises, les démarches de gestion des compétences ont pour ambition d'insuffler de la mobilité, de la flexibilité, de la réactivité et de la créativité, pour contribuer au décloisonnement et au changement. Chez Microtek, c'est plutôt l'inverse : paradoxalement, la gestion des compétences est orientée davantage vers la structuration et la standardisation, avec la création ou la mobilisation de plusieurs outils tels que le référentiel de compétences pour les opérateurs, les définitions de fonction, les profils de compétences, les chartes de formation, le support d'entretien d'évaluation... 
Pour autant, cette formalisation de la gestion des compétences n'est pas totalement achevée. Dans les faits, il subsiste des pratiques ou des principes qui entrent en tension avec les actions de formalisation: il n'y a pas de gestion prévisionnelle des emplois et des compétences au sens strict du terme, les organigrammes de remplacement n'existent pas, des promotions «maison» ont toujours cours, la notion d'autonomie figure dans la définition de la compétence, les dirigeants veulent recréer le «bouillon »... En d'autres termes, des principes culturels toujours en vigueur et des pratiques s'opposent aux efforts de formalisation.

Un deuxième point de discussion relie la croissance et le contenu de la gestion des compétences. Il nous semble, à cet égard, qu'il n'existe pas de déterminisme absolu de la croissance de la taille de l'entreprise pour rendre compte des modalités pratiques de la gestion des compétences au sein de Microtek. Certes, l'augmentation des effectifs et du volume des activités jouent un rôle évident dans la formalisation des actions observées ici et là, mais en aucune façon ce n'est le facteur structurant unique. D'autres éléments explicatifs sont à l'œuvre. Les différences de sensibilité entre les acteurs principaux sur la manière de gérer les compétences rendent compte également des pratiques constatées : les dirigeants, les responsables du département ressources humaines, les membres de l'encadrement, les salariés exercent chacun une influence et ont une idée assez précise de la direction à privilégier dans ce domaine. Dans cette liste, on retrouve les trois principaux acteurs identifiés par Flamholtz dans l'analyse des processus de management des transitions : les dirigeants, les membres de la fonction ressources humaines et l'encadrement (Flamholtz, 1995, mais aussi Mahé de Boislandelle, 1998b).

Une troisième piste de discussion nous renvoie à la question des compétences collectives. Celle-ci ne fait pas l'objet d'actions spécifiques de la part des managers de Microtek. Les salariés eux-mêmes ont du mal à cerner le contenu possible de cette notion, comme en témoigne cette déclaration d'un ingénieur : «Les compétences collectives, j'ai du mal à les percevoir. » Ce même ingénieur aurait plutôt tendance à évoquer le partage de valeurs communes, en d'autres mots, la culture de l'entreprise. Cette remarque rejoint les résultats d'une recherche américaine dans laquelle les dirigeants et fondateurs de petites et moyennes entreprises déclarent qu'ils recherchent avant tout des salariés qui épousent les valeurs de l'entreprise, ses croyances, ses intérêts plutôt que de s'assurer qu'ils possèdent bien les compétences requises par le fonctionnement de l'entreprise ou par les emplois (Heneman, Tansky et Camp, 2000). Ce constat d'absence d'actions portant spécifiquement sur la compétence collective n'est pas forcément problématique pour l'entreprise à l'heure actuelle, mais il pourrait le devenir : de plus en plus, les opérateurs devront être en mesure de relier leur action avec les opérations réalisées en amont et en aval. Dès à présent, il semble donc important de réfléchir à cette dimension collective. 
Un dernier point de discussion, enfin, touche au modèle d'analyse pertinent pour rendre compte de la gestion des ressources humaines dans ce type de PME à très forte évolution. Le mode de gestion des compétences chez Microtek s'inscrit à notre sens dans ce que les spécialistes des ressources humaines (Brabet, 1993) appellent « le modèle de la gestion des contradictions », qui s'oppose à deux autres modèles : «le modèle instrumental» et celui de «l'arbitrage managérial». Le modèle instrumental repose sur le postulat d'une convergence fondamentale des intérêts, des enjeux et des finalités, des différents acteurs aboutissant à l'égalité : efficacité économique égale efficacité sociale. Le modèle de l'arbitrage managérial met en relief les dimensions politiques de l'organisation, des zones de convergence mais aussi de divergence entre les intérêts des acteurs. Cette divergence reconnue est cependant conçue comme parfaitement réductible dans le long terme. Le modèle de la gestion des contradictions remet profondément en question les harmonies que supposent les deux autres modèles. Il affirme les enjeux contradictoires et ambivalents des acteurs, en négociation permanente et non réductibles à terme. Les actions expérimentales sont privilégiées : «Le management est [...] un processus expérimental visant à découvrir ce corps de connaissance qui, éventuellement, permet le contrôle. Les managers font des choix expérimentaux dans les stades de transition. Ils se débrouillent et ils apprennent. Le management des crises demande du self-control plutôt que du contrôle, une attitude de facilitation plutôt que de contrôle » (Van Gallen, cité par Brabet, 1993).

Au total, en paraphrasant Mahé de Boislandelle (1998b), on peut dire que « la gestion des compétences dans les PME en croissance n'est pas simple ». Devant cette complexité, notre contribution ne cherche pas à établir un modèle type mais avant tout à examiner, analyser et comprendre comment concrètement une PME en forte croissance a mis en œuvre une gestion des compétences, dans quelles conditions, avec quels résultats et quels en sont les éléments les plus structurants ou déterminants. Avec cette posture, nous sommes clairement dans le courant de la diversité et de la contingence, «la PME-champ» et non pas la «PME-objet» (Torrès, 1998). Cette posture de recherche dans les PME - et non sur les PME nécessite la multiplication des études de cas : c'est la raison pour laquelle nous avons entrepris un travail du même type dans une autre PME du secteur informatique, elle-même engagée dans une dynamique de développement très accentué. Il sera alors intéressant de confronter les observations et de prolonger les analyses menées au sein de Microtek.

\section{Bibliographie}

Aubret, J., P. GILBERT et F. PigeYre (2002), Management des compétences. Réalisations, concepts, analyses, Paris, Dunod. 
BAYAD, M. et D. NeBenhaus (1996), «Préoccupations de GRH et profil des dirigeants de PME », Économie et Sociétés, série Sciences de Gestion, vol. 10, nº 22, p. 185-203.

Bayad, M., H. Mahé de Boislandelle, D. Nebenhaus et P. SARnin (1995), «Paradoxe et spécificités des problématiques de gestion des ressources humaines en petites et moyennes entreprises », Gestion 2000, n 1, p. 95-108.

BRABET, J. (1993), « La gestion des ressources humaines en trois modèles », dans J. Brabet (dir.), Repenser la gestion des ressources humaines?, Paris, Economica, p. 69-141.

Brouwers, I. et al. (1997), Management humain et contexte de changement. Pour une approche constructiviste, Bruxelles, De Boeck Université.

ChurChiLl, N.C. et V.L. LeWIS (1983), «The five stages of small business growth », Harvard Business Review, mai-juin, p. 30-50.

D’Amboise, G. et D. GARAND (1995), «Identification des difficultés et besoins des PME en matière de gestion des ressources humaines », Gestion 2000, vol. 11, nº 1, p. 109-32.

DefÉliX C., M. Dubois et D. Retour (1999), «La gestion des compétences en PME: configurations et modalités de pilotage », Actes du Xe congrès de l'AGRH, Lyon, 9-10 septembre, p. 403-412.

DURAND, T. (2000), «L'alchimie de la compétence », Revue française de gestion, n 127 , janvier-février, p. 84-102.

FlAMHOLTZ, E. (1995), «Managing organizational transitions : implications for corporate and human resource management », European Management Journal, vol. 13, n 1 , mars, p. 39-51.

GARAND, D. et B. FABI (1994), «La conservation des ressources humaines en PME», Revue internationale PME, vol. 7, n ${ }^{\circ} 1$, p. 85-26.

GÉNIAUX, I. et S. MIRA BONNARDEL (2001), « La gestion des connaissances et des compétences dans les petites entreprises technologiques : une approche empirique », Revue internationale PME, vol. 14, no 2, p. 41-64.

GIRARD, B. (1997), «Pourquoi les PME restent-elles des PME?», Gérer et comprendre, Annales des Mines, septembre, p. 33-42.

GREINER, L. (1977), « Evolution and revolution as organizations grow », Harvard Business Review, vol. 50, no 4, p. 37-46, publié en français sous le titre «De l'utilité des crises dans l'entreprise », Harvard-L'Expansion, été, p. 22-29.

Hanks, S.H., C.J. WAtson, E. Jansen et G.N. Chandler (1993), «Tightening the life-cycle construct: a taxonomic study of growth stage configurations in hightechnology organizations », Entrepreneurship Theory and Practice, vol. 18, n 2, hiver, p. 5-22.

HENEMAN, R., J. TANSKY et M. CAMP (2000), «Human resource management practices in small and medium-sized enterprises: answered questions and future research perspectives », Entrepreneurship Theory and Practice, automne, vol. 25, no 1 , p. 11-26.

JOLLY, D. et L. RoCHE (1999), «La GRH dans les entreprises à fort contenu technologique », Management et conjoncture sociale, $\mathrm{n}^{\circ}$ 556, 10 mai, p. 25-32.

Revue internationale P.M.E., vol. 16, nos 3-4, 2003

(C) 2004 - Presses de l'Université du Québec

Édifice Le Delta I, 2875, boul. Laurier, bureau 450, Sainte-Foy, Québec G1V 2M2 • Tél. : (418) 657-4399 - www.puq.ca

Tiré de : Revue internationale P.M.E., vol. 16, nos 3-4, sous la direction de Pierre-André Julien • PME1603N Tous droits de reproduction, de traduction et d'adaptation réservés 
KaliKa, M. (1988), Structures d'entreprises. Réalité, déterminants, performance, Paris, Economica.

KATZ, J.A., H.E. ALDRICH, T.M. WelbouRnE et P.M. Williams (2000), « Guest editor's comment: special issue on human resource management and the SME : toward a new synthesis », Entrepreneurship Theory and Practice, vol. 25, nº 1, p. 7-10.

LERAY, Y. et A. JOYEAU (1999), «Gestion des emplois - compétences et gestion de la structure organisationnelle: l'expérience d'un établissement bancaire de taille moyenne», Gestion 2000, p. 137-157.

MaHÉ De Boislandelle, H. (1998a), Gestion des ressources humaines dans les PME, $2^{\mathrm{e}}$ édition, Paris, Economica.

MahÉ de Boislandelle, H. (1998b), «GRH en PME: universalité et contingences: essai de théorisation », Revue internationale PME, vol. 11, nos 2-3, p. 11-29.

NARO, G. (1990), «Les PME face à la gestion de leurs effectifs : comment adapter les ressources humaines aux impératifs stratégiques?», Revue internationale PME, vol. 3 , n ${ }^{\circ} 1$, p. $57-74$.

Nebenhaus, D. et P. SARnin (1993), «Problématiques et spécificités des recherches de GRH dans les PME», Actes du IVe congrès de l'AGRH, Georges Trépo (dir.), Association francophone de gestion des ressources humaines, p. 502-505.

PARADAS, A. (1998), «Évaluer la formation dans des PME françaises: résultats d'une étude empirique », Revue internationale PME, vol. 11, ${ }^{\text {os }}$ 2-3, p. 31-48.

Pettigrew, A.M. (1985), The Awakening Giant. Continuity and Change in Imperial Chemical Industries, Oxford / New York, Blackwell.

Pichault, F. et J. Nizet (2000), Les pratiques de gestion des ressources humaines, Paris, Seuil.

SCOUARNEC, A. (1999), «De la gestion de la compétence dans l'entreprise de taille moyenne : enjeux et limites », Gestion 2000, nº 6, novembre-décembre, p. 119-135.

STRATEGOR (1985), Stratégie, structure, décision, identité, Paris.

TORRÈs, O. (1998), «Vingt-cinq ans de recherche en PME : une discipline entre courants et contr|e-courants », dans O. Torrès (dir.), PME. De nouvelles approches, Paris, Economica, p. 17-53. 